\title{
Mycological profile of intertrigo of foot in a tertiary care hospital, Mysore, South India
}

\author{
Raghavendra Rao M${ }^{1}$, Vinay Kumar $V^{2}$, Satya Sai B ${ }^{3}$, Krishna Karthik $\mathbf{M}^{4}$, Ranjitha Shankare Gowda ${ }^{5, *}$ \\ ${ }^{1}$ Associate Professor, ${ }^{2}$ Tutor, ${ }^{3,4}$ Tutor and Research Scholar, ${ }^{5}$ Assistant Professor, Dept. of Microbiology, ${ }^{1,3,4,5}$ JSS Medical \\ College and Hospital, Mysore, Karnataka, ${ }^{2}$ Maheshwara Medical College and Hospital, Hyderabad, Telangana, India
}

*Corresponding Author: Ranjitha Shankare Gowda

Email: ranju.vish85@gmail.com

Received: $10^{\text {th }}$ October, 2018

Accepted: $23^{\text {rd }}$ November, 2018

\begin{abstract}
Introduction: Intertrigo is an inflammatory condition of skin folds commonly seen in any age from infancy to old age and one of the major cause for clinical consultations. Intertrigo is due to heat, moisture or lack of air circulation and the infection is most commonly seen in patients with diabetes. Foot intertrigo is the infection of Toe web spaces occurring mainly at interdigital toe web spaces. The friction of skin folds may lead to several complications which includes bacterial or fungal infections.

Materials and Methods: A total of 75 clinically suspected cases of foot intertrigo were included in the study. Skin scrapings from the suspected cases of intertrigo were collected with standard precautions and all the samples were subjected to direct microscopy using 10\% Potassium hydroxide and observed for presence of fungal elements and simultaneously the sample is processed for culture onto fungal culture media to identify the causative agents of foot intertrigo.

Results: A total of 75 skin scrapings from the infected foot for Mycological study were collected among which 38 samples were collected from male patients and 37 samples were from female patients. Of 75 skin scrapings, 31 were $10 \% \mathrm{KOH}$ positive and remaining 44 were negative to microscopy. On culture 34 samples were culture positive and 41 were culture negative. Out of 34 culture positive samples, Candida albicans were isolated in 15 specimens and Non-candida albicans in 6. Remaining samples yielded growth of moulds in which Dermatophytes (Trichophyton-3, Epidermophyton-1) were 4 isolates and Fusarium yielded growth in 9 samples.

Conclusion: From our study, we conclude that intertrigo is a chronic inflammation. This might occur at any age. In our descriptive study, Candida albicans were predominantly isolated followed by other moulds such as Fusarium and Dermatophytes.
\end{abstract}

Keywords: Intertrigo, Candida albicans, Fusarium, Dermatophytes.

\section{Introduction}

Intertrigo is an inflammatory rash of the superficial skin that usually occurs within skin folds. These folds are likely more prone to irritation. Factors such as moisture, friction and exposure to body secretions such as sweat, urine or feces may promote skin breakdown and are responsible for subsequent infections. This is a chronic inflammatory condition most commonly seen in diabetic patients. Environmental factors such as hot and humid weather play an important role in causing intertrigo. ${ }^{1}$

Foot intertrigo is the inflammation of Toe web spaces which often occurs in the 4th interdigital space extending to the dorsal and plantar aspects it is characterized primarily by mild erythema. ${ }^{2}$ Patients usually present with symptoms such as itching, burning, pain, swelling of the toes which is associated with difficulty in walking. Intertrigo can be clinically diagnosed with appropriate history and detailed physical examination by medical professionals. Differential diagnosis of intertrigo may include erythramas, inverse psoriasis, candidiasis and pyoderma etc. $^{3}$

Mycological agents responsible for intertrigo of foot may include yeasts, moulds, and dermatophytes, among which Candida spp are most common etiological agents reported so far in association with intertrigo. Intertrigo is usually misdiagnosed as dermatophytic infection. Proliferation of the organism are usually encouraged which complicates interdigital intertrigo. Certain yeasts such as Candia spp are also commonly associated with interdigital intertrigo and seborrheic dermatitis. ${ }^{4}$

Damage to the stratum corneum by the etiological agents will produce certain natural substances which are rich in antibiotic properties that usually encourages the multiplication or proliferation of antibiotic resistant strains. Removal of moisture prior to treatment is generally advised to maintain the skin integrity. Topical and systemic antifungals remains to be the mainstay in treatment of intertrigo if the etiological agents are known. Broad-spectrum antifungals that are usually used in treatment of superficial fungal infections belong to azole class. ${ }^{5}$ Considering this, the study was undertaken in a tertiary care hospital to find out the mycological agents causing intertrigo of foot. Little evidence-based literature supports any specific preventive measures for intertrigo. ${ }^{14}$ However, optimal prevention includes minimizing skin-on-skin friction, reducing heat and moisture around skinfolds, and keeping high-risk areas clean and dry. ${ }^{14}$ Patients should be warned about heat, humidity, and outside activities. 
Physical exercise usually is desirable, but patients should shower after exercise and keep intertriginous areas thoroughly dry. Wearing open-toed shoes may help prevent toe web intertrigo.

\section{Materials and Methods}

This is a hospital based prospective study done in the department of Microbiology, JSS Medical college and Hospital a tertiary care hospital situated in Mysore, South India. Samples were collected from the patients with clinical suspicion of intertrigo of foot. Detailed history such as age, sex, demographic and socioeconomic status of the patients were collected prior to sample collection. Clinical data of the patients was collected from the case records to rule out systemic illness.

Sample Collection: Skin scrapings were collected under the supervision of the professionals in the department of Dermatology by following standard precautions. Specimens were wrapped in clean black coloured paper and labelled with the patient's details including the site from where the samples have been collected. The same were transported to the department of Microbiology for laboratory investigations. The samples were divided into two portions: one for microscopic examination and one for culture.

\section{Direct Microscopy and Culture}

Samples that are received in the Microbiology department were processed for direct microscopy using $10 \%$ and $20 \%$ potassium hydroxide $(\mathrm{KOH})$ wet-mount examination, and screened for the presence of fungal elements, Simultaneously the other portion of the sample were inoculated on to Potato dextrose agar (PDA), Sabouraud dextrose agar (SDA), Sabouraud dextrose agar with antibiotics (SDAac) and incubated at room temperature for 2-4 weeks and observed for the growth. Isolated fungi were identified by macroscopic appearance and microscopic morphology using gram stain for yeasts and Lacotophenol Cotton Blue (LPCB) stainand slide culture for moulds. Germ tube test was performed to differentiate between Candida albicans and Non-albicans species.

\section{Results}

A Total of 75 skin scrapings were included from the clinically diagnosed cases of intertrigo of foot for mycological study which were received in the department of Microbiology during the study period. Of 75 samples collected 38 samples were collected from males and 37 samples were from female patients. Majority of cases i.e., $60(80 \%)$ cases belonged to the age group of 51-60 years. Among our study group 27 day-old neonate was youngest patient and 80 year old patient oldest. Occupational history of the patients revealed that majority of the patients i.e $30 \%$ of them were housewives followed by $26 \%$ were farmers who works in agricultural fields. Clinical picture showed that $60 \%$ of the patients presented with duration of infection for more than 60 days followed by $28 \%$ with duration of 41-50 days and 12\% with duration of 11-21 days. Skin scrapings were subjected simultaneously to direct microscopic examination using $10 \%$ or $20 \%$ potassium hydroxide and cultured on to fungal culture media for isolation of fungus. In total of 75 samples 34 samples yielded growth in which 31 were $\mathrm{KOH}$ positive and 3 were $\mathrm{KOH}$ negative (Table 1) and remaining 41 samples were both $\mathrm{KOH} \&$ culture negative (Table 1). Out of 34 cultures positive samples, yeasts growth was observed in 21 samples, in which Candida albicans were $15(44.1 \%)$ isolates and Non-candida albicans were $6(17.6 \%)$ isolates remaining samples yielded growth of moulds in which Dermatophytes (Trichophyton species-3, Epidermophyton-1) were $4(11.7 \%)$ isolates and Fusarium yielded growth in 9(26.4\%) samples (Table 2).

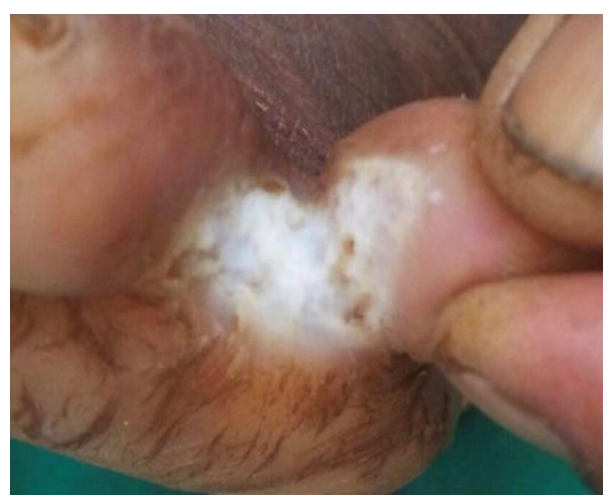

Fig. 1: $1^{\text {st }}$ Toe web space

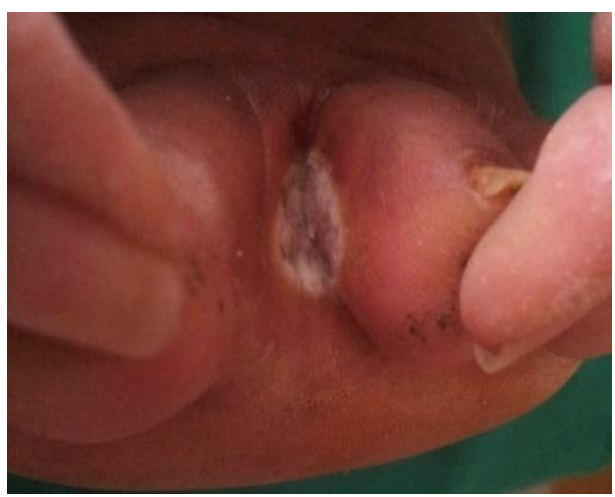

Fig. 2: $5^{\text {th }}$ Toe web space

Table 1: A comparision of both $\mathrm{KOH}$ microscopy and culture

\begin{tabular}{|c|c|c|c|}
\hline $\begin{array}{c}\text { Total Samples } \\
\text { (75) }\end{array}$ & $\begin{array}{c}\text { KOH } \\
\text { Positive }\end{array}$ & $\begin{array}{c}\text { KOH } \\
\text { Negative }\end{array}$ & Total \\
\hline Culture Positive & 31 & 03 & 34 \\
\hline Culture Negative & 0 & 41 & 41 \\
\hline
\end{tabular}




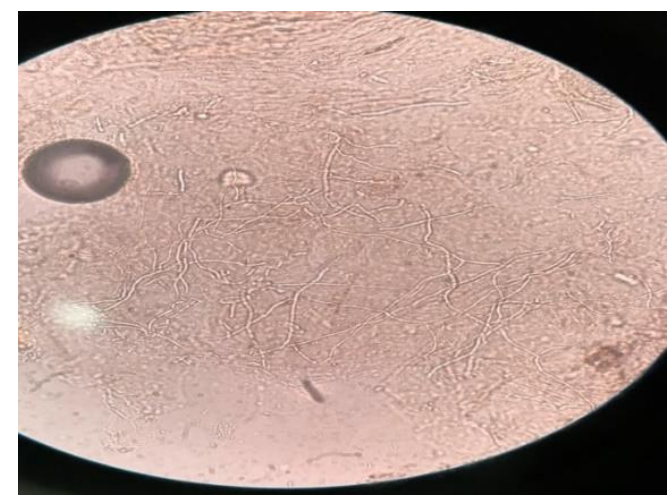

Fig. 3: Branched septate Hyphae (KOH)

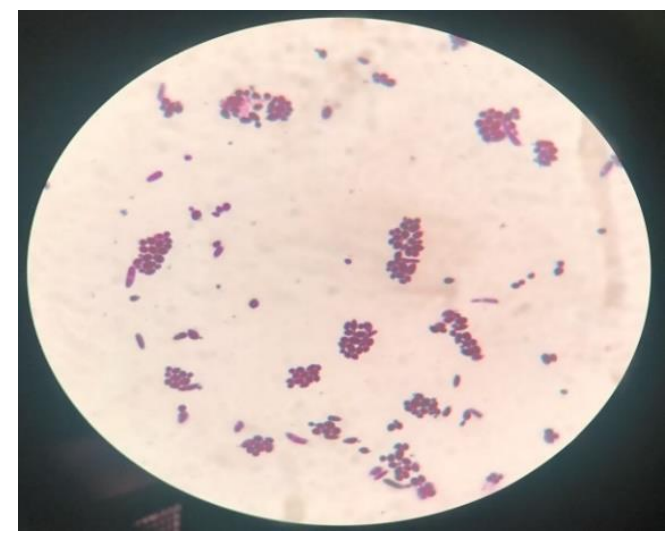

Fig. 4: Yeast cells (Grams stain)

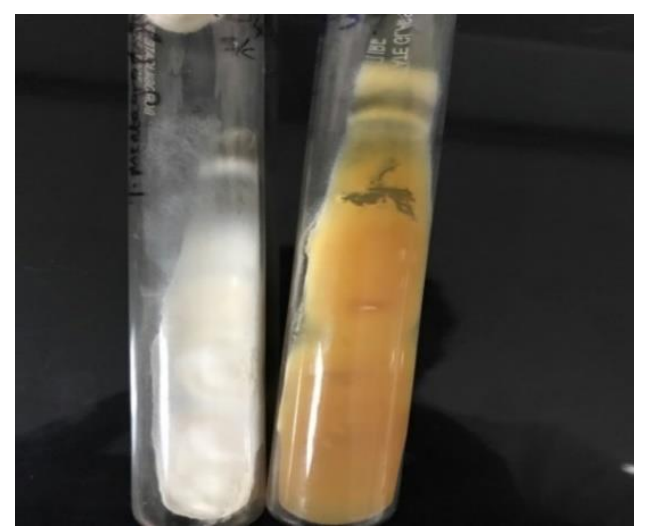

Fig. 5: Positive culture in SDA (moulds)

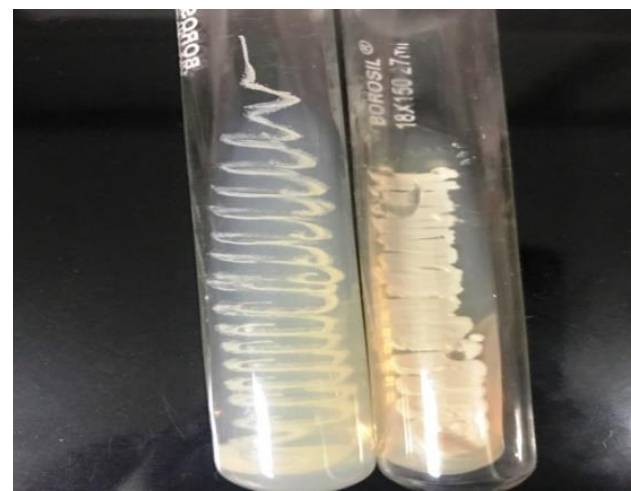

Fig. 6: Positive culture in SDA(yeast)
Table 2: Isolated fungi

\begin{tabular}{|l|c|c|}
\hline Isolates & Number & Percentage \\
\hline Candida albicans & 15 & $44.1 \%$ \\
\hline Non-albicans & 6 & $17.6 \%$ \\
\hline Dermatophytes & 4 & $11.7 \%$ \\
\hline Fusarium species & 9 & $26.4 \%$ \\
\hline Total & 34 & $100 \%$ \\
\hline
\end{tabular}

\section{Discussion}

It has been well documented that earth is natural territory for fungi and almost one fifth of world population suffers from mycoses. ${ }^{13}$ The usual clinical presentation of intertrigo of foot is presented as chronic erythematous skin peeling eruptions. This is often misdiagnosed as dermatophytoses or dermatitis associated with eczema. Few patients who don't respond to treatment with antifungals can be look into other conditions causing similar presentation. Clinical presentation of Intertrigo usually starts with erythematous area along with breakdown of the skin. This later converts as scaling which is more commonly seen at skin folds. Edges of the scales may contain pustules which is commonly associated with intense itching and pain when there is significant skin breakdown. These pustules are also called as satellite lesions which appears as red spots or small blister like. Selection of an appropriate treatment at this stage might reduce severe complications.

Out of 75pus samples 38 [50.67\%] samples were collected from male patients and 37 [49.33\%] samples were collected from female patients. In concordance a study conducted by Ahmad et al. ${ }^{6}$, reported higher rate in males (56.7\%). Majority of patients belong to the age group of $41-50(28 \%) .{ }^{6}$ Whereas in a study by Aste et al. ${ }^{7}$, found that foot intertrigo was common in the age group of 36-81 years, 19-74 years, and 12-48 years, respectively The age variations signifies that intertrigo is a common disorder that can affect any individual from infancy to old age. In a study by Aste et al., toe web spaces were involved bilaterally in the majority $(86.67 \%)$ of patients ${ }^{7}$, which was in concordance with our study. The reason for the toe web space infection might be majority of males are noticed as agriculturists and females are who engaged in household work in our study group, which is an important predisposing factor for toe web intertrigo. This is in concordance with the study of Ahmad et al. ${ }^{6}$ in which bilateral 4th and 5th toe web space were involved in majority $(41.76 \%)$ of patients in their study. In our study, the predominant organism isolated was Candida, which accounted for $44.1 \%$. This is consistent with the studies of Ahmad et al., and Kates SG., which showed $57.7 \%, 60 \%$, cases of Candida, respectively. ${ }^{3,6}$ The predominance of Candida in our study might be due to retention of moisture which can be more commonly seen in housewives, Agriculturists. Retention of moisture can increase the temperature and causes maceration in body folds. This is the most common problem, which is 
usually seen during summer and monsoon. Other predisposing factors along with climatic conditions also play a major role in this clinical condition.

\section{Conclusion}

Intertrigo is one of the chronic condition commonly observed in housewives even though it can be affect both sexes at any age. In the present study $4^{\text {th }}$ and $5^{\text {th }}$ toe web space being more commonly involved and caused by Candida albicans, Fusarium followed by Candida non-albicans and Dermatophytes. Therefore it is necessary to accurately identify causative agent of intertrigo to select appropriate antimicrobial agent which helps in prevention of complications.

\section{References}

1. Intertrigo and Common Secondary Skin Infections. CAmI A K. J Anniger, M.D. New Jersey Medical School, Newark, New Jersey. American Family Physician. 2005;72(5):833-8. www.aafp.org/afp

2. Kates SG, Nordstrom KM, McGinley KJ, Leyden JJ. Microbial ecology of interdigital infections of toe web spaces. J Am Acad Dermatol. 1990;22:578-82.2.

3. Neubert U, Braun-Falco O. Maceration of the interdigital spaces and gram negative infection of feet. Hautarzt. 1976;27:538-43.

4. Hope YM, Clayton YM, Hay RJ, Noble WC, Elder-Smith JG. Foot infection incoal miners: a reassessment. $\mathrm{Br} \mathrm{J}$ Dermatol. 1985;112:405-13.

5. Ahmad S, Am an S, Hussain I, Haroon TS. A clinicoetiological study of toe web fungal infection. J Pak Ass Dermatol. 2003;13:62-6.

6. Aste N, Atzori L, Zucca M, Pau M, Biggio P. Gramnegative bacterial toe web infection: a survey of 123 cases from the district of Cagliari, Italy. J Am Acad Dermatol. 2001;45:537-41.
7. Janniger CK, Schwartz RA, Szepietowski JC, Reich A. Intertrigo and common secondary skin infections. $A m$ Fam Physician. 2005;72:833-8.

8. Chapman MS, Brown JM, Linowski GJ: $0.1 \%$ tacrolimus ointment for the treatment of intertrigo. Arch Dermatol. 2005;141:787.

9. Spector JA, Singh SP, Karp NS: Outcomes after breast reduction - Does sizereally matter? Annals of Plastic Surgery. 2008;60:505-509.

10. J.-Y. Lin, Y.-L. Shih, and H.-C. Ho, "Foot bacterial intertrigo mimicking interdigital Tinea pedis," Chang Gung Medical Journal., 2011;34(1):44-49.

11. O. M. Rashid, J. C. Schaum, L. G. Wolfe, N. K. Brinster, and J. P. Neifeld. Prognostic variables and surgical management of foot melanoma review of a 25-year institutional experience. ISRN Dermatology, vol. 2011, Article ID 384729,8 pages, 2011.

12. Raghavendra Rao Morubagal, Rashmi Padmanabha Mahale, Sowmya Govindanahalli Shivappa, Tejashree Anantharajaurs and Madhuri Kulkarni. Mycological study of dermatophytosis in a part of South India., 2017;13:1-5.

13. Mistiaen P, Poot E, Hickox S, Jochems C, Wagner C. Preventing and treating intertrigo in the large skin folds of adults: a literature overview. Dermatol Nurs. 2004;16:43-6,49-57.

How to cite this article: Raghavendra R. M, Vinay K. V, Satya S. B, Krishna K. M, Gowda R S. Mycological profile of intertrigo of foot in a tertiary care hospital, Mysore, South India. Indian J Microbiol Res. 2018;5(4):504-507. 